\title{
Solution of Singularly Perturbed Differential-Difference Equations with Mixed Shifts Using Galerkin Method with Exponential Fitting
}

\author{
D. Kumara Swamy, ${ }^{1}$ K. Phaneendra, ${ }^{2}$ and Y. N. Reddy ${ }^{1}$ \\ ${ }^{1}$ Department of Mathematics, National Institute of Technology, Warangal, India \\ ${ }^{2}$ Department of Mathematics, University College of Science, Saifabad, Osmania University, Hyderabad, India \\ Correspondence should be addressed to K. Phaneendra; kollojuphaneendra@yahoo.co.in
}

Received 21 April 2016; Revised 3 August 2016; Accepted 8 September 2016

Academic Editor: Chuanzhi Bai

Copyright (C) 2016 D. Kumara Swamy et al. This is an open access article distributed under the Creative Commons Attribution License, which permits unrestricted use, distribution, and reproduction in any medium, provided the original work is properly cited.

Galerkin method is presented to solve singularly perturbed differential-difference equations with delay and advanced shifts using fitting factor. In the numerical treatment of such type of problems, Taylor's approximation is used to tackle the terms containing small shifts. A fitting factor in the Galerkin scheme is introduced which takes care of the rapid changes that occur in the boundary layer. This fitting factor is obtained from the asymptotic solution of singular perturbations. Thomas algorithm is used to solve the tridiagonal system of the fitted Galerkin method. The method is analysed for convergence. Several numerical examples are solved and compared to demonstrate the applicability of the method. Graphs are plotted for the solutions of these problems to illustrate the effect of small shifts on the boundary layer solution.

\section{Introduction}

Singularly perturbed differential-difference equations (SPDDEs) arise very frequently in the mathematical modelling of real life situations in science and engineering [1-3]. In the mathematical modelling of a physical system as in control theory, the presence of small time parasitic parameters like moments of inertia, resistances, inductances, and capacitances increases the order and stiffness of these systems. The suppression of these small constants results in the reduction of the order of the system. Such systems are termed as singular perturbation systems and when these systems take into account the past history as well as the present state of the physical system then they are called singularly perturbed delay differential equations. Delay differential equations arise in first-exit time problems in neurobiology and in mathematical formulation of various practical phenomena in biosciences. A differential-difference equation with the presence of shift terms induces large amplitudes and exhibits oscillations, resonance, turning point behaviour, and boundary and interior layers. Hence, to control such behaviour, we need some simple and efficient numerical techniques.

Lange and Miura [3-7] published a series of papers extending the method of matched asymptotic expansions initially developed for ordinary differential equations to obtain approximate solution of singularly perturbed differentialdifference equations.

Numerical analysis of singularly perturbed differentialdifference turning point problems was initiated by Kadalbajoo and Sharma. In a series of papers, [8-10], they gave many robust numerical techniques for the solution of such type of problems. Kadalbajoo and Sharma [8] elucidate a numerical method to solve boundary value problems for singularly perturbed differential-difference equation with mixed shifts. Kadalbajoo and Sharma [9] proposed a numerical method to solve boundary value problems for a singularly perturbed differential-difference equation of a mixed type, that is, which contains both types of terms having negative shifts as well as positive shifts, and considered the case in which the solution of the problem exhibits rapid oscillations. Kadalbajoo and Sharma [10] described a numerical approach based on finite 
difference method to solve a mathematical model arising from a model of neuronal variability. Kadalbajoo and Kumar [11] used B-spline collocation method with fitted mesh for the solution of singularly perturbed differential-difference equations with small delay.

Patidar and Sharma [12] combined fitted-operator methods with Micken's nonstandard finite difference techniques for the numerical approximations of singularly perturbed linear delay differential equations. Kadalbajoo et al. [13] derived $\varepsilon$-uniformly convergent fitted methods for the solution of singularly perturbed differential-difference equation (SPDDE). Kumar and Sharma [14] presented a numerical scheme based on B-spline collocation to approximate the solution of boundary value problems for singularly perturbed differential-difference equations with delay and advance.

With this motivation, an exponentially fitting factor is introduced in Galerkin method for the solution of singularly perturbed differential-difference equation with delay and advanced parameters. In Section 2, description of the problem is given. In Section 3, numerical scheme for the solution of the problem is presented and Section 4 deals with convergence analysis of the proposed scheme. To demonstrate the efficiency of the proposed method, numerical experiments are carried out for several test problems and the results are given in Section 5. Finally the conclusions are given in the last section.

\section{Description of the Problem}

Consider a linear singularly perturbed differential-difference equation of the following form:

$$
\begin{aligned}
& \varepsilon y^{\prime \prime}(x)+p(x) y^{\prime}(x)+q(x) y(x-\delta)+r(x) y(x) \\
& +s(x) y(x+\eta)=f(x)
\end{aligned}
$$

on $(0,1)$, under the boundary conditions

$$
\begin{aligned}
& y(x)=\varphi(x) \quad \text { on }-\delta \leq x \leq 0, \\
& y(1)=\gamma(x) \quad \text { on } 1 \leq x \leq 1+\eta .
\end{aligned}
$$

Here $\varepsilon$ is a small parameter such that $0<\varepsilon \ll 1, p(x)$, $q(x), r(x), s(x), f(x), \phi(x)$, and $\gamma(x)$ are smooth functions, and $0<\delta=o(\varepsilon), 0<\eta=o(\varepsilon)$ are, respectively, the delay (negative shift) and the advance (positive shift) parameters. If $p(x)-\delta q(x)+\eta s(x)>0$, the solution of (1) with (2) exhibits layer at the left end of the interval and if $p(x)-\delta q(x)+\eta s(x)<$ 0 , the layer exists at the right end of the interval.

Since the solution $y(x)$ of boundary value problem equations (1) and (2) is sufficiently differentiable, we expand the terms $y(x-\delta)$ and $y(x+\eta)$ using Taylor series; we get

$$
\begin{aligned}
& y(x-\delta) \approx y(x)-\delta y^{\prime}(x)+O\left(\delta^{2}\right) \\
& y(x+\eta) \approx y(x)+\eta y^{\prime}(x)+O\left(\eta^{2}\right) .
\end{aligned}
$$

Using (3) and (4) in (1), we get

$$
\begin{aligned}
& \varepsilon y^{\prime \prime}(x)+a(x) y^{\prime}(x)+b(x) y(x) \\
& =f(x)+O\left(\delta^{2}+\eta^{2}\right) .
\end{aligned}
$$

Equation (5) is an asymptotically equivalent second-order singular perturbation problem of (1) with boundary conditions

$$
\begin{aligned}
& y(0)=\varphi(0), \\
& y(1)=\gamma(1) .
\end{aligned}
$$

Since $0<\delta \ll 1$ and $0<\eta \ll 1$, the transition from (1) to (5) is admitted. This replacement is significant from the computational point of view. For more details on the validity of this transition, one can refer El'sgol'ts and Norkin [15]. Thus, the solution of (5) provides a good approximation to the solution of (1).

Here,

$$
\begin{aligned}
& a(x)=p(x)-\delta q(x)+\eta s(x), \\
& b(x)=q(x)+r(x)+s(x) .
\end{aligned}
$$

\section{Numerical Scheme}

3.1. Left-End Boundary Layer Problem. Let $0=x_{0}, x_{1}, \ldots$, $x_{N}=1$ be a decomposition of the considered interval $[0,1]$ into $N$ equal intervals with constant mesh length $h$. Then we have the nodes $x_{i}=i h$, for $0,1, \ldots, N$. Assume that $a(x), b(x)$, and $f(x)$ are sufficiently continuously differentiable functions in $[0,1]$. If $a(x) \geq M>0$ in $[0,1]$ where $M$ is a positive constant, (5) has a unique solution $y(x)$ which, in general, displays a boundary layer of width $O(\varepsilon)$ at $x=0$.

Lemma 1 (Doolan et al. [16] and O'Malley [17]). Let $\tilde{y}(x)=$ $y_{0}+z_{0}$ be the zeroth-order asymptotic approximation to the solution of (5), where $y_{0}$ represents the zeroth-order approximate outer solution (i.e., the solution of the reduced problem of (5)) and $z_{0}$ represents the zeroth-order approximate solution in the boundary layer region of (5).

Then for a fixed positive integer $i$,

$$
\begin{aligned}
& \lim _{h \rightarrow 0} y(i h) \approx y_{0}(0)+\left(\varphi(0)-y_{0}(0)\right) e^{-a(0) i \rho} \\
& \text { where } \rho=\frac{h}{\varepsilon} .
\end{aligned}
$$

Proof. Let $y_{0}(x)$ be the solution of the reduced problem of (5)

$$
p(x) y_{0}^{\prime}(x)+q(x) y_{0}(x)=f(x), \quad y_{0}(1)=\gamma(1)
$$


and $z_{0}(t)$ is the solution of the boundary value problem (cf. O’Malley [17])

$$
\begin{aligned}
z_{0}^{\prime \prime}(t)+p(0) z_{0}^{\prime}(t) & =0, \\
z_{0}(0) & =\varphi(0)-y_{0}(0), \\
z_{0}(\infty) & =0
\end{aligned}
$$

$$
\text { where } t=\frac{x}{\varepsilon} \text {. }
$$

From the theory of singular perturbation, the zerothorder asymptotic approximation to the solution of (4) is (cf. O’Malley [17])

$$
\tilde{y}(x) \approx y_{0}(x)+\frac{a(0)}{a(x)}\left(\varphi(0)-y_{0}(0)\right) e^{-\int_{0}^{x}(a(x) / \varepsilon) d x} .
$$

As we are considering the differential equations on sufficiently small subintervals, the coefficients could be assumed to be locally constant. Hence,

$$
\tilde{y}(x) \approx y_{0}(x)+\left(\varphi(0)-y_{0}(0)\right) e^{-(a(0) / \varepsilon) x} .
$$

So, at the nodal points, we have

$$
\begin{aligned}
\tilde{y}\left(x_{i}\right) \approx y_{0}\left(x_{i}\right)+\left(\varphi(0)-y_{0}(0)\right) e^{(a(0) / \varepsilon) x_{i}} & \\
& i=0,1, \ldots, N ;
\end{aligned}
$$

that is,

$$
\widetilde{y}(i h) \approx y_{0}(i h)+\left(\varphi(0)-y_{0}(0)\right) e^{-(a(0) / \varepsilon) i h} .
$$

Therefore

$$
\begin{array}{r}
\lim _{h \rightarrow 0} \tilde{y}(i h) \approx y_{0}(0)+\left(\varphi(0)-y_{0}(0)\right) \exp \{-a(0) i \rho\} \\
\text { for } i=0,1,2,3, \ldots, N,
\end{array}
$$

where $\rho=h / \varepsilon$.

Now, we consider the difference scheme [18] by Galerkin method as follows:

Select a set of basis functions $\varphi_{j}(x), j=0,1, \ldots, N$, which will define an interpolation scheme for the approximate solution over a grid of points $a=x_{0}<x_{1}<$ $\cdots<x_{N+1}=b$. For simplicity, we use piecewise Lagrange polynomials $l_{i}(x)$ of first degree as the basis functions. These interpolating polynomials are

$$
\begin{aligned}
& l_{0}(\xi)=\frac{\xi-\xi_{1}}{\xi_{0}-\xi_{1}}=\frac{(1-\xi)}{2} \\
& l_{1}(\xi)=\frac{\xi-\xi_{0}}{\xi_{1}-\xi_{0}}=\frac{(1+\xi)}{2}
\end{aligned}
$$

in local element coordinates $-1 \leq \xi \leq 1$.

The $N$ nodal values of the approximate solution $y$ at the interior nodes $x_{1}, x_{2}, \ldots, x_{N}$ are determined using this basis. The given boundary conditions determine the value of $y(x)$ at the end nodes $x_{0}$ and $x_{N+1}$. The Galerkin method is now employed to obtain the integral equations; we have

$$
\begin{gathered}
\left(\varepsilon y^{\prime \prime}(x)+a(x) y^{\prime}(x)+b(x) y(x), \varphi_{j}\right) \\
=\left(f(x), \varphi_{j}\right) \quad \text { for } j=1,2, \ldots, N
\end{gathered}
$$

which is an integral equation $\int_{x_{0}}^{x_{N}}\left(\varepsilon y^{\prime \prime}(x)+a(x) y^{\prime}(x)+\right.$ $b(x) y(x)) \phi_{j} d x=\int_{x_{0}}^{x_{N}} f(x) \varphi_{j} d x$.

Since $y$ is sum of piecewise linear Lagrange polynomials, the second-order derivatives appearing in (17) vanish except at the element boundaries $x_{i}$, where they become infinite.

By integration by parts, (18) becomes

$$
\begin{gathered}
-\left(\varepsilon \frac{d y}{d x}, \frac{d \phi_{j}}{d x}\right)+\left(a(x) \frac{d y}{d x}+b(x) y, \phi_{j}\right) \\
+\left(\varepsilon \frac{d y}{d x} \phi_{j}\right)_{a}^{b}=\left(f(x), \phi_{j}\right) .
\end{gathered}
$$

Using the substitution of trial function $y(x)=\phi_{0}(x)+$ $\sum_{i=1}^{N} y_{i} \phi_{j}(x)$ into the integral equation (19), we have

$$
\begin{aligned}
\sum_{i=1}^{N} y_{i}\left(\varepsilon \frac{d \phi_{i}}{d x}, \frac{d \phi_{j}}{d x}\right)-\sum_{i=1}^{N} y_{i}\left(a(x) \frac{d \phi_{i}}{d x}+b(x) \phi_{i}, \phi_{j}\right) \\
=-\alpha\left(\frac{d l_{0}}{d x}, \frac{d \phi_{j}}{d x}\right)-\beta\left(\frac{d l_{N+1}}{d x}, \frac{d \phi_{j}}{d x}\right) \\
+\alpha\left(a(x) \frac{d l_{0}}{d x}+b(x) l_{0}, \phi_{j}\right) \\
+\beta\left(a(x) \frac{d l_{N+1}}{d x}+b(x) l_{N+1}, \phi_{j}\right) \\
+\left(\varepsilon \frac{d y}{d x} \phi_{j}\right)_{a}^{b}-\left(f(x), \phi_{j}\right)
\end{aligned}
$$

for $j=1,2, \ldots, N$.

It can be observed that all quantities on the right side of (20) can be computed from known boundary data to obtain $N$ equations in the $N$ unknown values $y_{i}$ at the interior nodes. The integrals in (20) can be solved by taking advantage of local coordinate $(\xi)$ system.

Since

$$
\begin{aligned}
x & =\frac{(b-a)}{2} \xi+\frac{(b+a)}{2}=\frac{h}{2} \xi+\frac{(b+a)}{2}, \\
\xi & =\frac{2}{h}\left(x-\frac{(b+a)}{2}\right) \Longrightarrow \\
\frac{d \xi}{d x} & =\frac{2}{h}
\end{aligned}
$$


we have, by simple integration,

$$
\begin{aligned}
& \int_{a}^{b} \frac{d \phi_{i}}{d x} \frac{d \phi_{j}}{d x} d x=\int_{-1}^{1} \frac{d l_{0}}{d \xi} \frac{d \xi}{d x} \frac{d l_{1}}{d \xi} \frac{d \xi}{d x} \frac{h}{2} d \xi=\frac{-1}{h}, \\
& \text { for } i=j-1, \\
& \int_{a}^{b} \frac{d \phi_{i}}{d x} \frac{d \phi_{j}}{d x} d x=\int_{-1}^{1} \frac{d l_{1}}{d \xi} \frac{d \xi}{d x} \frac{d l_{0}}{d \xi} \frac{d \xi}{d x} \frac{h}{2} d \xi=\frac{-1}{h}, \\
& \int_{a}^{b} \frac{d \phi_{i}}{d x} \frac{d \phi_{j}}{d x} d x=\int_{-1}^{1} \frac{d l_{1}}{d \xi} \frac{d \xi}{d x} \frac{d l_{1}}{d \xi} \frac{d \xi}{d x} \frac{h}{2} d \xi=\frac{1}{h}, \\
& \int_{a}^{b} \frac{d \phi_{i}}{d x} \frac{d \phi_{j}}{d x} d x=0 \quad \text { for }|i-j|>1 .
\end{aligned}
$$

By assuming $a(x), b(x)$, and $f(x)$ as constants, the integral equation (20) gives, for a typical internal node $j$,

$$
\begin{gathered}
y_{j-1}\left(\frac{-\varepsilon}{h}+\frac{a}{2}-\frac{b h}{6}\right)+y_{j}\left(\frac{2 \varepsilon}{h}-\frac{2 b h}{3}\right) \\
+y_{j+1}\left(\frac{-\varepsilon}{h}-\frac{a}{2}-\frac{b h}{6}\right)=-f_{j} h .
\end{gathered}
$$

Equation (23), when rearranged, gives the following system of difference equations:

$$
\begin{aligned}
& \varepsilon\left(\frac{y_{j+1}-2 y_{j}+y_{j-1}}{h^{2}}\right)+a\left(\frac{y_{j+1}-y_{j-1}}{2 h}\right) \\
& +b\left(\frac{y_{j-1}+4 y_{j}+y_{j+1}}{6}\right)= \\
& \quad f_{j} ; \\
& \quad \text { for } 1 \leq j \leq N-1 .
\end{aligned}
$$

Now, introduce a fitting factor $\sigma$ in the Galerkin scheme as follows:

$$
\begin{gathered}
\sigma \varepsilon\left(\frac{y_{j+1}-2 y_{j}+y_{j-1}}{h^{2}}\right)+a_{j}\left(\frac{y_{j+1}-y_{j-1}}{2 h}\right) \\
+b_{j}\left(\frac{y_{j-1}+4 y_{j}+y_{j+1}}{6}\right)=f_{j}
\end{gathered}
$$

for $1 \leq j \leq N-1$ with $y_{0}=\phi(0), y_{N}=\gamma(1)$. Here $\sigma$ is a fitting factor which is to be determined in such a way that the solution of (25) converges uniformly to the solution of (5). Multiplying (25) by $h$ and taking the limit as $h \rightarrow 0$ (in [16]), we get

$$
\begin{aligned}
& \lim _{h \rightarrow 0}\left(\frac{\sigma}{\rho}\left(y_{j+1}-2 y_{j}+y_{j-1}\right)+\frac{1}{2} a_{j}\left(y_{j+1}-y_{j-1}\right)\right) \\
& \quad=0 \quad \text { if } f_{j}-b y_{j} \text { is bounded } \\
& \therefore \lim _{h \rightarrow 0}\left(\frac{\sigma}{\rho}(y(j h+h)-2 y(j h)+y(j h-h))\right. \\
& \left.\quad+\frac{1}{2} a_{j}(y(j h+h)-y(j h-h))\right)=0 .
\end{aligned}
$$

Now, approximating the solution $y(x)$ by zeroth-order asymptotic approximation $\tilde{y}(x)$ and using Lemma 1 , we have

$$
\begin{aligned}
\lim _{h \rightarrow 0} \tilde{y}(i h)= & y_{0}(0)+\left(\varphi(0)-y_{0}(0)\right) e^{-a(0) i \rho}, \\
\lim _{h \rightarrow 0} \tilde{y}(j h+h)= & y_{0}(0) \\
& +\left(\varphi(0)-y_{0}(0)\right) e^{-a(0) j \rho} e^{-a(0) \rho}, \\
\lim _{h \rightarrow 0} \tilde{y}(j h-h)= & y_{0}(0) \\
& +\left(\varphi(0)-y_{0}(0)\right) e^{-a(0) j \rho} e^{a(0) \rho} .
\end{aligned}
$$

Using the above equations in (27), we get

$$
\sigma=\frac{\rho}{2} a(0) \operatorname{coth}\left[\left(\frac{a^{2}(0)-\varepsilon b(0)}{a(0)}\right) \frac{\rho}{2}\right] .
$$

From (25), we have

$$
\begin{gathered}
y_{j-1}\left(\frac{\varepsilon \sigma}{h^{2}}-\frac{a_{j}}{2 h}+\frac{b_{j}}{6}\right)-y_{j}\left(\frac{2 \varepsilon \sigma}{h^{2}}-\frac{2 b_{j}}{3}\right) \\
+y_{j+1}\left(\frac{\varepsilon \sigma}{h^{2}}+\frac{a_{j}}{2 h}+\frac{b_{j}}{6}\right)=f_{j}
\end{gathered}
$$

for $j=1,2, \ldots, N-1$.

Equation (30) can be written as a three-term recurrence relation as follows:

$$
E_{j} y_{j-1}-F_{j} y_{i}+G_{j} y_{j+1}=H_{j} ; \quad j=1,2, \ldots, N-1,
$$

where

$$
\begin{aligned}
& E_{j}=\left(\frac{\varepsilon \sigma}{h^{2}}-\frac{a_{j}}{2 h}+\frac{b_{j}}{6}\right), \\
& F_{j}=\left(\frac{2 \varepsilon \sigma}{h^{2}}-\frac{2 b_{j}}{3}\right), \\
& G_{j}=\left(\frac{\varepsilon \sigma}{h^{2}}+\frac{a_{j}}{2 h}+\frac{b_{j}}{6}\right), \\
& H_{j}=f_{j} .
\end{aligned}
$$

The tridiagonal system equation (31) is solved using Thomas algorithm.

3.2. Right-End Layer Problems. We now discuss the method for singularly perturbed two-point boundary value problems with right-end boundary layer of the underlying interval. Assume that $a(x), b(x)$, and $f(x)$ are sufficiently continuously differentiable functions in $[0,1]$. Furthermore, assume that $a(x) \leq M<0$ in $[0,1]$, where $M$ is a negative constant. Under these assumptions, (5) has a unique solution $y(x)$ which, in general, displays a boundary layer of width $O(\varepsilon)$ at $x=1$.

Lemma 2. Let $y(x)=y_{0}+z_{0}$ be the zeroth-order asymptotic approximation to the solution of (5), where $y_{0}$ represents the 
zeroth-order approximate outer solution and $z_{0}$ represents the zeroth-order approximate solution in the boundary layer region.

Then for a fixed positive integer $i$,

$$
\begin{aligned}
\lim _{h \rightarrow 0} y(i h) \approx & y_{0}(0) \\
+\left(\beta-y_{0}(1)\right) \exp \left\{a(1)\left(\frac{1}{\varepsilon}-i \rho\right)\right\} & \text { where } \rho=\frac{h}{\varepsilon} .
\end{aligned}
$$

Proof. The proof is based on asymptotic analysis (Doolan et al. [16] and O'Malley [17]) and is similar to the proof of Lemma 1.

Applying the same procedure as in Section 3 and using Lemma 2, we get the tridiagonal system equation (20) with fitting factor as

$$
\sigma=\frac{\rho}{2} a(0) \operatorname{coth}\left[\left(\frac{a^{2}(1)-\varepsilon b(1)}{a(1)}\right) \frac{\rho}{2}\right] .
$$

\section{Convergence Analysis}

Writing the tridiagonal system equation (31) in matrix-vector form, we get

$$
A Y=C
$$

in which $A=\left(m_{i j}\right), 1 \leq i, j \leq N-1$, is a tridiagonal matrix of order $N-1$, with

$$
\begin{aligned}
m_{i+1} & =\frac{\sigma \varepsilon}{h}-\frac{a_{i}}{2}+\frac{h b_{i}}{6}, \\
m_{i i} & =-\frac{2 \sigma \varepsilon}{h}-\frac{2 h b_{i}}{3} \\
m_{i-1} & =\frac{\sigma \varepsilon}{h}-\frac{a_{i}}{2}+\frac{h b_{i}}{6},
\end{aligned}
$$

and $C=\left(d_{i}\right)$ is a column vector with $d_{i}=h H_{i}$, where $i=$ $1,2, \ldots, N-1$ with local truncation error

$$
\tau_{i}=h^{2}\left[\frac{b_{i}}{6} y_{i}^{\prime \prime}\right]+O\left(h^{3}\right)
$$

and $Y=\left(y_{0}, y_{1}, \ldots, y_{N}\right)^{t}$.

We also have

$$
A \bar{Y}-T(h)=C
$$

where $\bar{Y}=\left(\overline{y_{0}}, \overline{y_{1}}, \ldots, \overline{y_{N}}\right)^{t}$ denotes the actual solution and $T(h)=\left(T_{0}\left(h_{0}\right), T_{1}\left(h_{1}\right), \ldots, T_{N}\left(h_{N}\right)\right)^{t}$ is the local truncation error.

From (35) and (38), we get

$$
A(\bar{Y}-Y)=T(h)
$$

Thus, the error equation is

$$
A E=T(h),
$$

where $E=\bar{Y}-Y=\left(e_{0}, e_{1}, \ldots, e_{N}\right)^{t}$.

Let the $i$ th row elements sum of matrix $A$ be $S_{i}$; then we have

$$
\begin{aligned}
& S_{i}=\sum_{j=1}^{N-1} m_{i j}=-\frac{\varepsilon \sigma}{h}+\frac{5 h b_{i}}{6}+\frac{a_{i}}{2}, \text { for } i=1, \\
& S_{i}=\sum_{j=1}^{N-1} m_{i j}=\sum_{j=1}^{N-1} m_{i j}=h b_{i}=B_{i_{0}}, \\
& \qquad \text { for } i=2,3, \ldots, N-2, \\
& S_{i}=\sum_{j=1}^{N-1} m_{i j}=-\frac{\sigma \varepsilon}{h}+\frac{5 h b_{i}}{6}-\frac{a_{i}}{2}, \text { for } i=N-1 .
\end{aligned}
$$

We can choose $h$ sufficiently small so that the matrix $A$ is irreducible and monotone. It follows that $A^{-1}$ exists and its elements are nonnegative.

Hence, from (40), we get

$$
\begin{gathered}
E=A^{-1} T(h), \\
\|E\| \leq\left\|A^{-1}\right\| \cdot\|T(h)\| .
\end{gathered}
$$

Also from the theory of matrices we have

$$
\sum_{i=1}^{N-1} \bar{m}_{k, i} S_{i}=1, \quad k=1(1) N-1,
$$

where $\bar{m}_{k, i}$ is $(k, i)$ element of the matrix $A^{-1}$ for some $i_{0}$ between 1 and $N-1$.

Therefore,

$$
\sum_{i=1}^{N-1} \bar{m}_{k, i} \leq \frac{1}{\min _{1 \leq i \leq N-1} S_{i}}=\frac{1}{B_{i_{o}}} \leq \frac{1}{\left|B_{i_{o}}\right|},
$$

where $B_{i_{0}}=h b_{i}$. We define $\left\|A^{-1}\right\|=\max _{1 \leq k \leq N-1} \sum_{i=1}^{N-1}\left|\bar{m}_{k, i}\right|$ and $\|T(h)\|=\max _{1 \leq i \leq N-1}\left|T_{i}(h)\right|$.

From (37), (40), (43), and (45), we get

$$
e_{j}=\sum_{i=1}^{N-1} \bar{m}_{k, i} T_{i}(h), \quad j=1(1) N-1
$$

which implies

$$
e_{j} \leq \frac{O\left(h^{2}\right)}{\left|B_{i_{o}}\right|}, \quad j=1(1) N-1 .
$$

Therefore,

$$
\|E\|=O(h)
$$

that is, our method reduces to a first-order convergent for uniform mesh. 
TABLE 1: The maximum absolute errors in solution of Example 1.

\begin{tabular}{|c|c|c|c|c|c|c|}
\hline$\varepsilon \downarrow$ & $N=8$ & $N=16$ & $N=32$ & $N=64$ & $N=128$ & $N=256$ \\
\hline \multicolumn{7}{|c|}{ Present method $\delta=0.5 \varepsilon, \eta=0.5 \varepsilon$} \\
\hline $10^{-1}$ & 0.03707062 & 0.01012487 & 0.00237565 & 0.0005848 & 0.00014565 & $3.6379 e-005$ \\
\hline $10^{-2}$ & 0.06876102 & 0.04407092 & 0.02342131 & 0.00865832 & 0.002129327 & 0.00050922 \\
\hline $10^{-3}$ & 0.08556830 & 0.05231609 & 0.02808039 & 0.01392938 & 0.00720360 & 0.00356728 \\
\hline $10^{-4}$ & 0.08740211 & 0.05431975 & 0.03006119 & 0.01590962 & 0.00810919 & 0.00401592 \\
\hline $10^{-5}$ & 0.08758721 & 0.05452232 & 0.03026147 & 0.01610995 & 0.00830856 & 0.00421487 \\
\hline $10^{-6}$ & 0.08760574 & 0.05454260 & 0.03028152 & 0.01613000 & 0.00832852 & 0.00423478 \\
\hline \multicolumn{7}{|c|}{ Results in Kadalbajoo and Sharma [10] } \\
\hline $10^{-1}$ & 0.12011566 & 0.07181396 & 0.04482982 & 0.02694612 & 0.01516093 & 0.00775036 \\
\hline $10^{-2}$ & 0.18727108 & 0.10697821 & 0.05904116 & 0.30796891 & 0.01567964 & 0.00799076 \\
\hline $10^{-3}$ & 0.20429729 & 0.11915028 & 0.06879232 & 0.03655236 & 0.01893849 & 0.00963304 \\
\hline $10^{-4}$ & 0.20614146 & 0.12048418 & 0.06989944 & 0.03721375 & 0.01932774 & 0.00984236 \\
\hline $10^{-5}$ & 0.20632746 & 0.12061888 & 0.07001167 & 0.03728089 & 0.01936732 & 0.00986365 \\
\hline $10^{-6}$ & 0.20634608 & 0.12063236 & 0.07002291 & 0.03728761 & 0.01937129 & 0.00986578 \\
\hline
\end{tabular}

\section{Numerical Examples}

To demonstrate the applicability of the method, we have applied the method on four boundary value problems. These examples have been chosen because they have been widely discussed in literature and exact solutions are available for comparison.

The exact solution of the boundary value problem

$$
\begin{aligned}
& \varepsilon y^{\prime \prime}(x)+p(x) y^{\prime}(x)+q(x) y(x-\delta)+r(x) y(x) \\
& +s(x) y(x+\eta)=f(x)
\end{aligned}
$$

under the boundary conditions

$$
\begin{aligned}
& y(x)=\varphi(x), \quad-\delta \leq x \leq 0, \\
& y(x)=\gamma(x), \quad 1 \leq x \leq 1+\eta,
\end{aligned}
$$

is

$$
y(x)=c_{1} e^{m_{1} x}+c_{2} e^{m_{2} x}+\frac{f}{c},
$$

where $c=(q+r+s), c_{1}=\left(-f+\gamma c+e^{m_{2}}(f-\phi c)\right) / c\left(e^{m_{1}}-e^{m_{2}}\right)$, $c_{2}=\left(f-\gamma c+e^{m_{1}}(-f+\phi c)\right) / c\left(e^{m_{1}}-e^{m_{2}}\right)$,

$$
\begin{aligned}
& m_{1}=\frac{-(p-q \delta+s \eta)+\sqrt{(p-q \delta+s \eta)^{2}-4 c \varepsilon}}{2 \varepsilon}, \\
& m_{2}=\frac{-(p-q \delta+s \eta)-\sqrt{(p-q \delta+s \eta)^{2}-4 c \varepsilon}}{2 \varepsilon} .
\end{aligned}
$$

Example 1. Consider the model boundary value problem of the type given by (1)-(2) having the boundary layer at the left end

$$
\varepsilon y^{\prime \prime}+y^{\prime}-2 y(x-\delta)-5 y+y(x+\eta)=0
$$

with boundary conditions $y(x)=1,-\delta \leq x \leq 0$, and $y(x)=$ $1,1 \leq x \leq 1+\eta$.

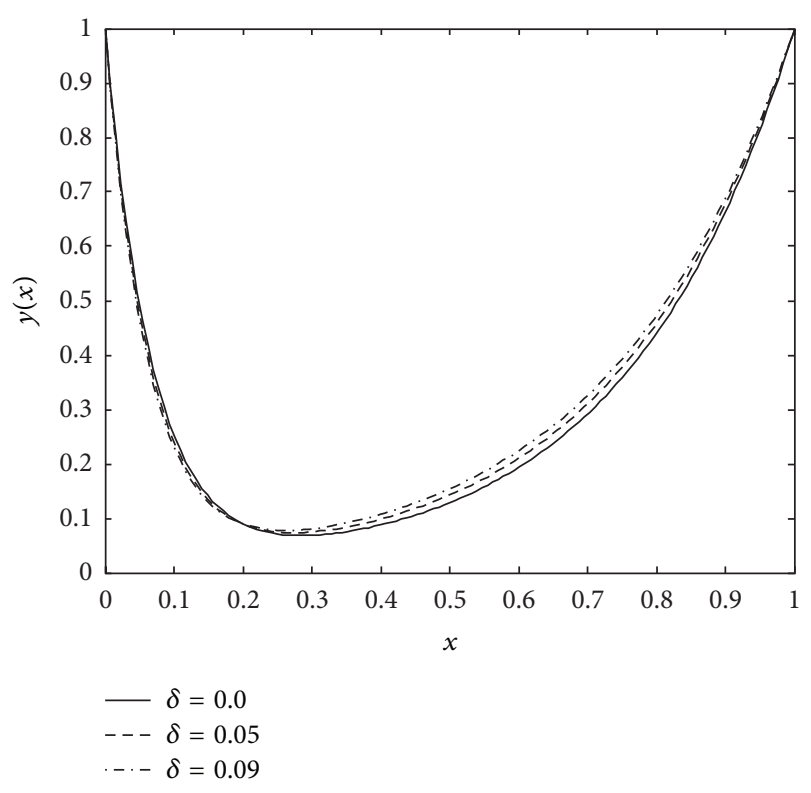

FIGURE 1: Numerical solution of Example 1 for different values of $\delta$ with $\varepsilon=0.1, \eta=0.05$.

The maximum absolute errors are given in Tables 1 and 2 for different values of the delay and advanced parameters with perturbation parameter. The effect of the small parameters on the boundary layer solutions is shown in Figures 1 and 2.

Example 2. Consider the boundary value problem having the boundary layer at the left end

$$
\varepsilon y^{\prime \prime}+y^{\prime}-2 y(x-\delta)+y-y(x+\eta)=-1
$$

with boundary conditions $y(x)=1,-\delta \leq x \leq 0$, and $y(x)=$ $1,1 \leq x \leq 1+\eta$.

The maximum absolute errors are given in Table 3 for different values of $\varepsilon$ with the delay and advance parameter 


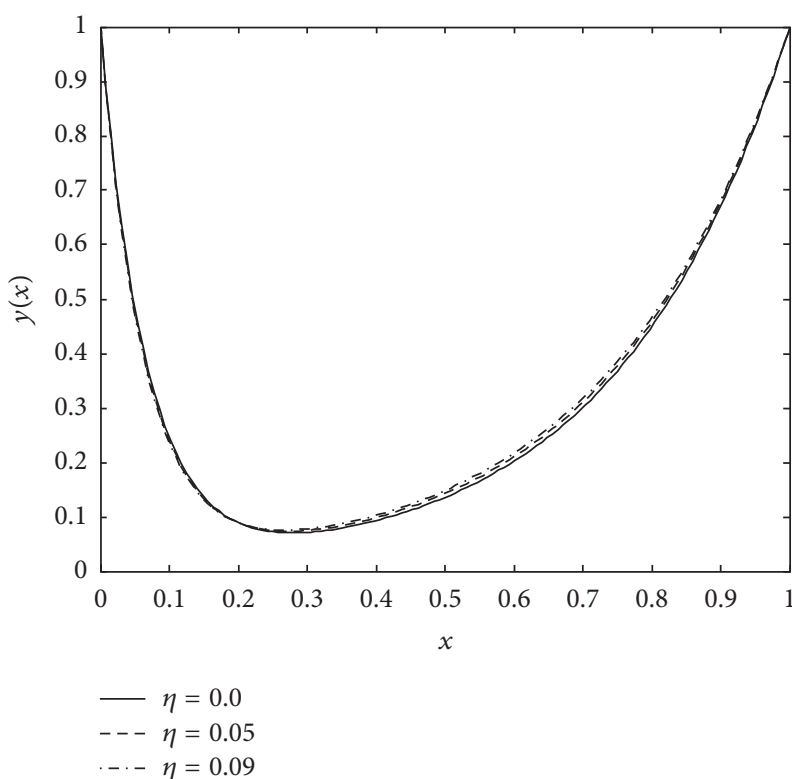

FIGURE 2: Numerical solution of Example 1 for different values of $\eta$ with $\varepsilon=0.1, \delta=0.05$.

TABLE 2: The maximum errors in solution of Example 1 with $\varepsilon=0.1$.

\begin{tabular}{|c|c|c|c|c|}
\hline & $N=8$ & $N=32$ & $N=128$ & $N=512$ \\
\hline \multicolumn{5}{|c|}{ Present method } \\
\hline$\delta \downarrow$ & \multicolumn{4}{|c|}{$\eta=0.5 \varepsilon$} \\
\hline 0.00 & 0.03724038 & 0.00231155 & 0.00014202 & $8.8820 e-006$ \\
\hline 0.05 & 0.03707062 & 0.00237565 & 0.00014565 & $9.0928 e-006$ \\
\hline 0.09 & 0.03669100 & 0.00241210 & 0.00014764 & $9.2248 e-006$ \\
\hline$\eta \downarrow$ & \multicolumn{4}{|c|}{$\delta=0.5 \varepsilon$} \\
\hline 0.00 & 0.03720017 & 0.00234619 & 0.00014399 & $8.9938 e-006$ \\
\hline 0.05 & 0.03707062 & 0.00237565 & 0.00014565 & $9.0928 e-006$ \\
\hline 0.09 & 0.03690625 & 0.00239551 & 0.00014675 & $9.1626 e-006$ \\
\hline \multicolumn{5}{|c|}{ Results in Kadalbajoo and Sharma [10] } \\
\hline$\delta \downarrow$ & \multicolumn{4}{|c|}{$\eta=0.5 \varepsilon$} \\
\hline 0.00 & 0.09190267 & 0.03453494 & 0.01164358 & 0.00300463 \\
\hline 0.05 & 0.10233615 & 0.03823132 & 0.01295871 & 0.00335137 \\
\hline 0.09 & 0.11018870 & 0.04110846 & 0.01400144 & 0.00362925 \\
\hline$\eta \downarrow$ & \multicolumn{4}{|c|}{$\delta=0.5 \varepsilon$} \\
\hline 0.00 & 0.09720079 & 0.03640446 & 0.01229476 & 0.00317786 \\
\hline 0.05 & 0.10233615 & 0.03823132 & 0.01295871 & 0.00335137 \\
\hline 0.09 & 0.10632014 & 0.03965833 & 0.01348348 & 0.00349050 \\
\hline
\end{tabular}

values $\delta=0.5 \varepsilon, \eta=0.5 \varepsilon$. The effect of the small parameters on the boundary layer solutions is shown in Figures 3 and 4 .

Example 3. Consider the boundary value problem of the type given by (1)-(2) having the boundary layer at the right end

$$
\varepsilon y^{\prime \prime}-y^{\prime}-2 y(x-\delta)+y-2 y(x+\eta)=0
$$

with boundary conditions $y(x)=1,-\delta \leq x \leq 0$, and $y(x)=$ $-1,1 \leq x \leq 1+\eta$.

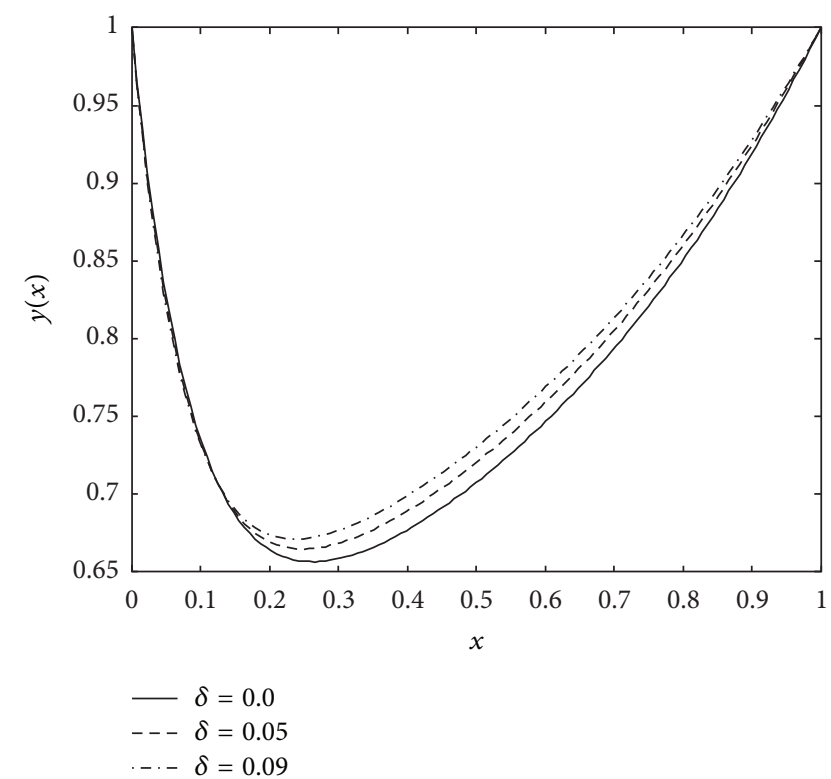

FIgURE 3: Numerical solution of Example 2 for different values of $\delta$ with $\varepsilon=0.1, \eta=0.05$.

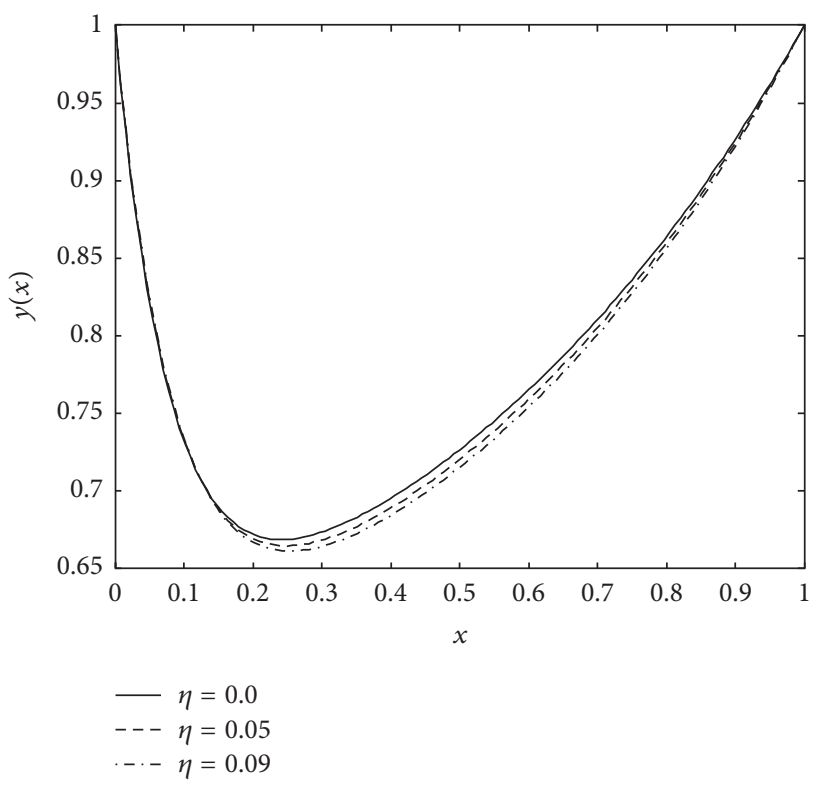

FIGURE 4: Numerical solution of Example 2 for different values of $\eta$ with $\varepsilon=0.1, \delta=0.05$.

The maximum absolute errors are given in Table 4 with $\varepsilon=0.1$ for different values of the delay and advance parameters. The effect of the small parameters on the boundary layer solutions is shown in Figures 5 and 6.

Example 4. Consider the boundary value problem having the boundary layer at the left end

$$
\varepsilon y^{\prime \prime}+0.5 y^{\prime}-3 y(x-\delta)-2 y+2 y(x+\eta)=1
$$

with boundary conditions $y(x)=1,-\delta \leq x \leq 0$, and $y(x)=$ $0,1 \leq x \leq 1+\eta$ 
TABLE 3: The maximum errors in solution of Example 2.

\begin{tabular}{|c|c|c|c|c|c|c|}
\hline$\varepsilon \downarrow$ & $N=8$ & $N=16$ & $N=32$ & $N=64$ & $N=128$ & $N=256$ \\
\hline \multicolumn{7}{|c|}{ Present method $\delta=0.5 \varepsilon, \eta=0.5 \varepsilon$} \\
\hline $10^{-1}$ & 0.00470784 & 0.00123359 & 0.00029344 & $7.2455 e-005$ & $1.8125 e-005$ & $4.5281 e-006$ \\
\hline $10^{-2}$ & 0.01585466 & 0.00697845 & 0.00240001 & 0.00093287 & 0.00024806 & $5.8741 e-005$ \\
\hline $10^{-3}$ & 0.01910533 & 0.01015879 & 0.00512445 & 0.00244082 & 0.00105444 & 0.00037664 \\
\hline $10^{-4}$ & 0.01944288 & 0.01049291 & 0.00545649 & 0.00277170 & 0.00138358 & 0.00067752 \\
\hline $10^{-5}$ & 0.01947677 & 0.01052645 & 0.00548983 & 0.00280492 & 0.00141674 & 0.00071065 \\
\hline $10^{-6}$ & 0.01948016 & 0.01052981 & 0.00549316 & 0.00280824 & 0.00142006 & 0.000713967 \\
\hline \multicolumn{7}{|c|}{ Results in Kadalbajoo and Sharma [10] } \\
\hline $10^{-1}$ & 0.08579690 & 0.05129568 & 0.03202130 & 0.01924723 & 0.01098354 & 0.00553597 \\
\hline $10^{-2}$ & 0.13376506 & 0.07641301 & 0.04217226 & 0.02199778 & 0.01119974 & 0.00570769 \\
\hline $10^{-3}$ & 0.14592663 & 0.08510734 & 0.04913737 & 0.02610883 & 0.01352749 & 0.00688074 \\
\hline $10^{-4}$ & 0.14724390 & 0.08606013 & 0.04992817 & 0.02658125 & 0.01380553 & 0.00703026 \\
\hline $10^{-5}$ & 0.14737676 & 0.08615634 & 0.05000834 & 0.02662921 & 0.01383380 & 0.00704546 \\
\hline $10^{-6}$ & 0.14739006 & 0.08616597 & 0.05001637 & 0.02663401 & 0.01383663 & 0.00704699 \\
\hline
\end{tabular}

TABLE 4: The maximum errors in solution of Example 3 with $\varepsilon=0.1$.

\begin{tabular}{|c|c|c|c|c|}
\hline & $N=8$ & $N=32$ & $N=128$ & $N=512$ \\
\hline \multicolumn{5}{|c|}{ Present method } \\
\hline$\delta \downarrow$ & \multicolumn{4}{|c|}{$\eta=0.5 \varepsilon$} \\
\hline 0.00 & 0.031377538 & 0.001800241 & 0.000112071 & $7.0036 e-006$ \\
\hline 0.05 & 0.029748010 & 0.001700026 & 0.000105418 & $6.5860 e-006$ \\
\hline 0.09 & 0.028294285 & 0.001611053 & $9.9793 e-005$ & $6.2344 e-006$ \\
\hline$\eta \downarrow$ & \multicolumn{4}{|c|}{$\delta=0.5 \varepsilon$} \\
\hline 0.00 & 0.027910529 & 0.001587651 & $9.8361 e-005$ & $6.1442 e-006$ \\
\hline 0.05 & 0.029748010 & 0.001700026 & 0.000105 & $6.5860 e-006$ \\
\hline & 0.031068500 & 0.001781207 & 0.000110800 & $6.9223 e-006$ \\
\hline \multicolumn{5}{|c|}{ Results in Kadalbajoo and Sharma [10] } \\
\hline$\delta \downarrow$ & \multicolumn{4}{|c|}{$\eta=0.5 \varepsilon$} \\
\hline 0.00 & 0.09930002 & 0.03685072 & 0.01331683 & 0.00342882 \\
\hline 0.05 & 0.09997296 & 0.03218424 & 0.01167102 & 0.00299572 \\
\hline 0.09 & 0.10044578 & 0.02850398 & 0.01038902 & 0.00266379 \\
\hline$\eta \downarrow$ & \multicolumn{4}{|c|}{$\delta=0.5 \varepsilon$} \\
\hline 0.00 & 0.10055269 & 0.02759534 & 0.01007834 & 0.00258299 \\
\hline 0.05 & 0.09997296 & 0.03218424 & 0.01167102 & 0.00299572 \\
\hline 0.09 & 0.09944067 & 0.03591410 & 0.01297367 & 0.00334044 \\
\hline
\end{tabular}

The maximum absolute errors are given in Table 5 and Table 6 for different values of delay and advanced parameters with perturbation parameter. The effect of the small parameters on the boundary layer solutions is shown in Figures 7 and 8 .

\section{Discussion and Conclusion}

An exponentially fitted Galerkin method has been presented for solving singularly perturbed differential-difference equations with delay as well as advance parameters. To demonstrate the applicability of the method, three examples with left-end and one with right-end boundary layer have been solved for different values of the delay, advance, and

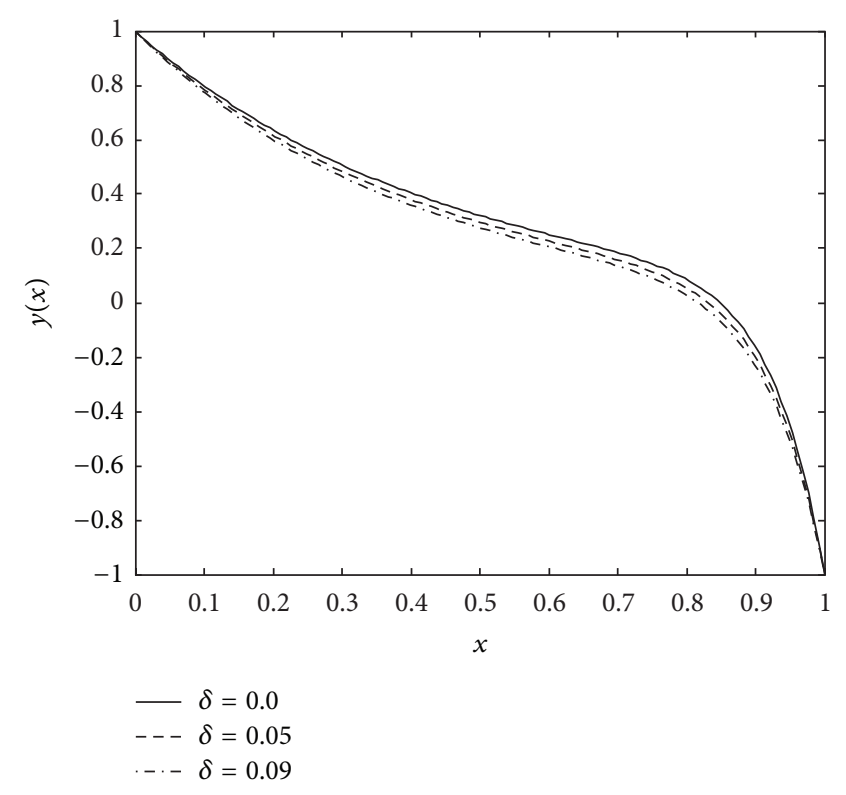

FIGURE 5: Numerical solution of Example 3 for different values of $\eta$ with $\varepsilon=0.1, \eta=0.05$.

perturbation parameters. The numerical results are taken by using MATLAB coding and solutions have been compared with the exact solutions and maximum absolute errors are presented in tables. To show the efficiency of the method, we have compared results of the proposed scheme with the results of Kadalbajoo and Sharma [10]. The rate of convergence in the examples is given in Table 7 . It is observed that the present method approximates the exact solution very well for which other classical finite difference methods fail to give good results. The effect of the delay and advance parameters on the solutions has also been investigated and presented by using graphs. When the solution of the boundary value problem exhibits layer behaviour on the left side, the effect of delay or advance on the solution in the boundary layer region 
TABLE 5: The maximum errors in solution of Example 4.

\begin{tabular}{ccccccc}
\hline$\varepsilon \downarrow$ & $N=8$ & $N=16$ & $N=32$ & $N=64$ & $N=128$ & $N=256$ \\
\hline \multicolumn{3}{c}{} & \multicolumn{3}{c}{$\delta=0.5 \varepsilon, \eta=0.5 \varepsilon$} \\
$10^{-1}$ & 0.02753382 & 0.00616170 & 0.00156771 & 0.00038927 & $9.7155 e-005$ & $2.4278 e-005$ \\
$10^{-2}$ & 0.09909124 & 0.05582849 & 0.02171656 & 0.00554645 & 0.00131747 & 0.00032522 \\
$10^{-3}$ & 0.10181648 & 0.06264442 & 0.03547474 & 0.01902508 & 0.00950250 & 0.00372083 \\
$10^{-4}$ & 0.10207612 & 0.06281638 & 0.03556178 & 0.01906740 & 0.00989895 & 0.00504914 \\
$10^{-5}$ & 0.10210236 & 0.06283382 & 0.03557070 & 0.01907016 & 0.00989794 & 0.00504604 \\
$10^{-6}$ & 0.10210499 & 0.06283557 & 0.03557159 & 0.01907044 & 0.00989798 & 0.00504673 \\
\hline
\end{tabular}

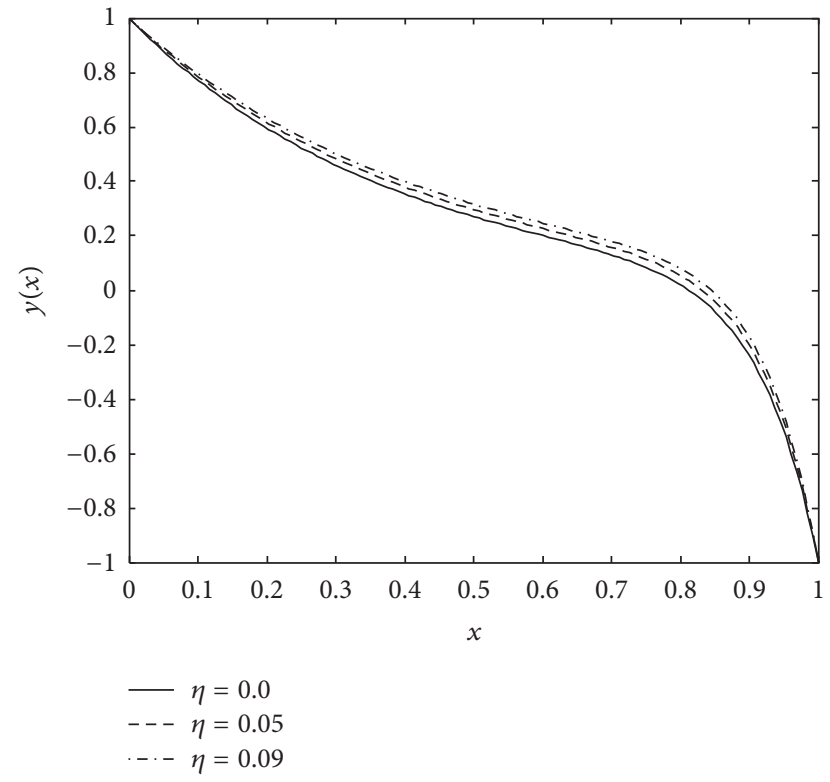

FIGURE 6: Numerical solution of Example 3 for different values of $\eta$ with $\varepsilon=0.1, \delta=0.05$.

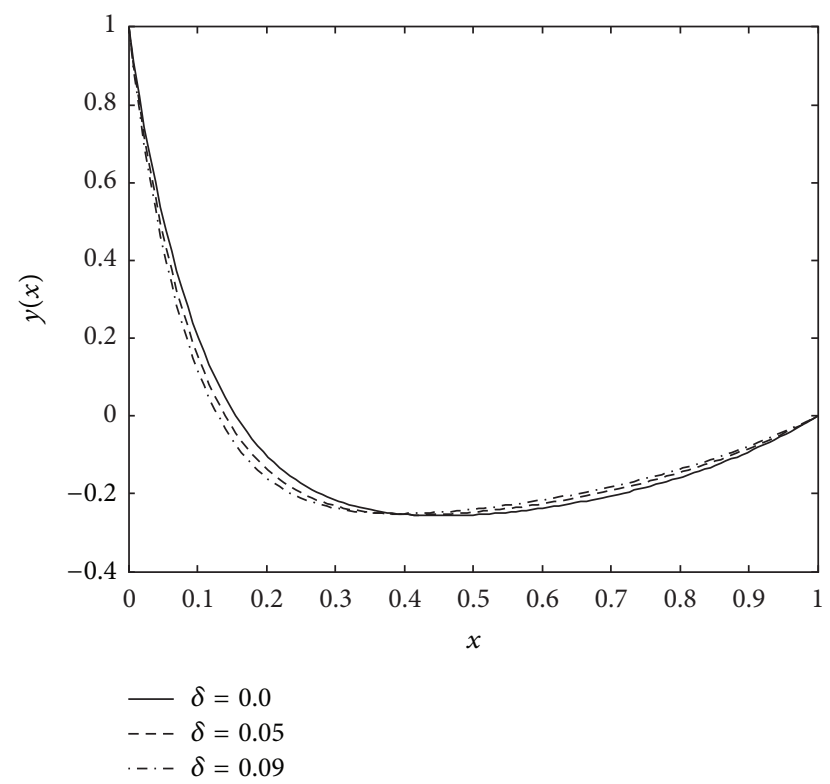

FIgURE 7: Numerical solution of Example 4 for different values of $\eta$ with $\varepsilon=0.1, \eta=0.05$.
TABLE 6: The maximum errors in solution of Example 4 with $\varepsilon=0.1$.

\begin{tabular}{|c|c|c|c|c|}
\hline & $N=8$ & $N=32$ & $N=128$ & $N=512$ \\
\hline$\delta \downarrow$ & \multicolumn{4}{|c|}{$\eta=0.5 \varepsilon$} \\
\hline 0.00 & 0.025347510 & 0.001425327 & $8.9204 e-005$ & $5.5742 e-006$ \\
\hline 0.05 & 0.027533826 & 0.001567710 & $9.7155 e-005$ & $6.0690 e-006$ \\
\hline 0.09 & 0.028669770 & 0.001645550 & 0.000102186 & $6.3826 e-006$ \\
\hline$\eta \downarrow$ & \multicolumn{4}{|c|}{$\delta=0.5 \varepsilon$} \\
\hline 0.00 & 0.026174618 & 0.001478341 & $9.2083 e-005$ & $5.7527 e-006$ \\
\hline 0.05 & 0.027533826 & 0.001567710 & $9.7155 e-005$ & $6.0690 e-006$ \\
\hline 0.09 & 0.028348272 & 0.001623113 & 0.00010057 & $6.2854 e-006$ \\
\hline
\end{tabular}

TABLE 7: The rate of convergence of the examples with $\delta=0.5 \varepsilon$ and $\eta=0.5 \varepsilon$.

\begin{tabular}{llcccc}
\hline$\varepsilon \downarrow$ & $N=8$ & $N=16$ & $N=32$ & $N=64$ & $N=128$ \\
\hline \multicolumn{5}{c}{ Example 1} \\
$10^{-2}$ & 0.6418 & 0.9120 & 1.4357 & 2.0237 & 2.0640 \\
$10^{-3}$ & 0.7098 & 0.8977 & 1.0114 & 0.9513 & 1.0139 \\
$10^{-4}$ & 0.6862 & 0.8536 & 0.9180 & 0.9723 & 1.0138 \\
$10^{-5}$ & 0.6839 & 0.8494 & 0.9095 & 0.9553 & 0.9791 \\
$10^{-6}$ & 0.6836 & 0.8489 & 0.9087 & 0.9536 & 0.9758 \\
\hline \multicolumn{5}{c}{ Example 2 } \\
$10^{-2}$ & 1.1839 & 1.5399 & 1.3633 & 1.9110 & 2.0782 \\
$10^{-3}$ & 0.9112 & 0.9873 & 1.0700 & 1.2109 & 1.4852 \\
$10^{-4}$ & 0.8898 & 0.9434 & 0.9772 & 1.0024 & 1.0301 \\
$10^{-5}$ & 0.8877 & 0.9392 & 0.9688 & 0.9854 & 0.9954 \\
$10^{-6}$ & 0.8875 & 0.9388 & 0.9680 & 0.9837 & 0.9920 \\
\hline \multicolumn{5}{c}{ Example 3 } \\
$10^{-2}$ & 1.1839 & 1.5398 & 1.3633 & 1.9112 & 2.0779 \\
$10^{-3}$ & 0.9112 & 0.9872 & 1.0700 & 1.2109 & 1.4853 \\
$10^{-4}$ & 0.8898 & 0.9433 & 0.9771 & 1.0024 & 1.0300 \\
$10^{-5}$ & 0.8877 & 0.9391 & 0.9688 & 0.9854 & 0.9954 \\
$10^{-6}$ & 0.8875 & 0.9387 & 0.9679 & 0.9837 & 0.9920 \\
\hline \multicolumn{5}{c}{ Example 4 } \\
$10^{-2}$ & 0.8278 & 1.3622 & 1.9692 & 2.0738 & 2.0183 \\
$10^{-3}$ & 0.7007 & 0.8204 & 0.8989 & 1.0015 & 1.3527 \\
$10^{-4}$ & 0.7004 & 0.8208 & 0.8992 & 0.9458 & 0.9712 \\
$10^{-5}$ & 0.7004 & 0.8209 & 0.8994 & 0.9461 & 0.9720 \\
$10^{-6}$ & 0.7004 & 0.8209 & 0.8994 & 0.9461 & 0.9718 \\
\hline & \multicolumn{5}{c}{. }
\end{tabular}

is negligible while, in the outer region, it is considerable and the change in the advance affects the solution in similar 


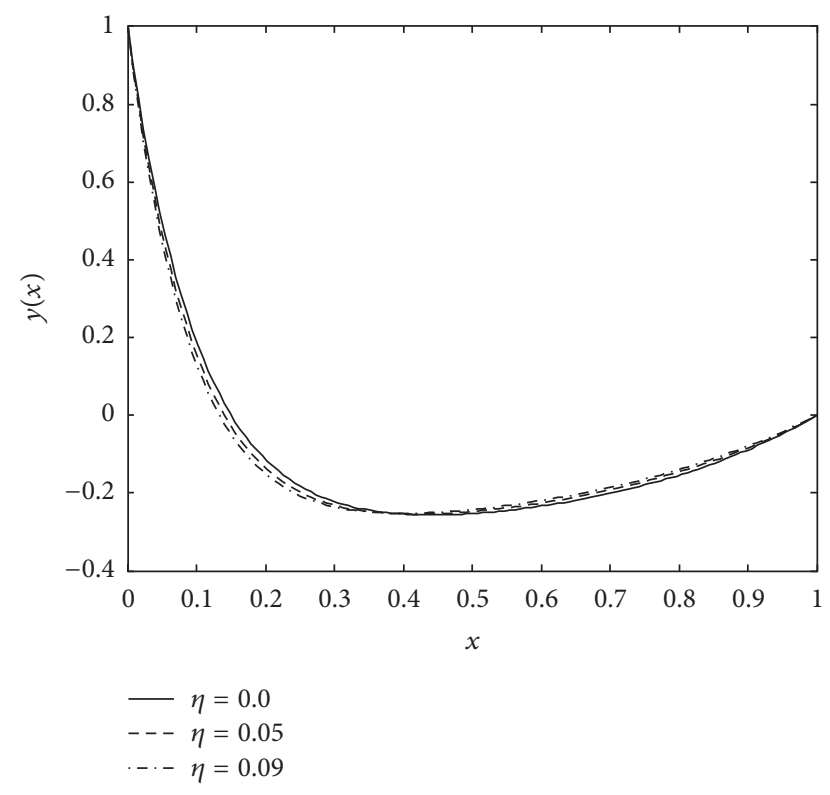

FIGURE 8: Numerical solution of Example 4 for different values of $\eta$ with $\varepsilon=0.1, \delta=0.05$.

fashion; that is, the width of the layer in the outer region increases as the advance parameter increases and decreases as the delay increases, but reversely (Figures 1-4). When the solution of the boundary value problem exhibits layer behaviour on the right side, the changes in delay or advance affect the solution in boundary layer region as well as outer region. The thickness of the layer increases as the size of the delay increases while it decreases as the size of the advance increases (Figures 5 and 6).

\section{Competing Interests}

The authors declare that they have no competing interests.

\section{References}

[1] M. W. Derstine, H. M. Gibbs, F. A. Hopf, and D. L. Kaplan, "Bifurcation gap in a hybrid optically bistable system," Physical Review A, vol. 26, no. 6, pp. 3720-3722, 1982.

[2] V. Y. Glizer, "Asymptotic analysis and solution of a finitehorizon $H_{\infty}$ control problem for singularly-perturbed linear systems with small state delay," Journal of Optimization Theory and Applications, vol. 117, no. 2, pp. 295-325, 2003.

[3] C. G. Lange and R. M. Miura, "Singular perturbation analysis of boundary-value problems for differential-difference equations. II. Rapid oscillations and resonances," SIAM Journal on Applied Mathematics, vol. 45, no. 5, pp. 687-707, 1985.

[4] C. G. Lange and R. M. Miura, "Singular perturbation analysis of boundary value problems for differential-difference equations III. Turning point problems," SIAM Journal on Applied Mathematics, vol. 45, no. 5, pp. 708-734, 1985.

[5] C. G. Lange and R. M. Miura, "Singular perturbation analysis of boundary value problems for differential-difference equations. V. Small shifts with layer behavior," SIAM Journal on Applied Mathematics, vol. 54, no. 1, pp. 249-272, 1994.
[6] C. G. Lange and R. M. Miura, "Singular perturbation analysis of boundary-value problems for differential-difference equations. VI. Small shifts with rapid oscillations," SIAM Journal on Applied Mathematics, vol. 54, no. 1, pp. 273-283, 1994.

[7] C. G. Lange and R. M. Miura, "Singular perturbation analysis of boundary value problems for differential-difference equations," SIAM Journal on Applied Mathematics, vol. 42, no. 3, pp. 502531, 1982.

[8] M. K. Kadalbajoo and K. K. Sharma, "Numerical analysis of boundary-value problems for singularly-perturbed differentialdifference equations with small shifts of mixed type," Journal of Optimization Theory and Applications, vol. 115, no. 1, pp. 145163, 2002.

[9] M. K. Kadalbajoo and K. K. Sharma, "Numerical analysis of boundary-value problems for singularly perturbed differentialdifference equations: small shifts of mixed type with rapid oscillations," Communications in Numerical Methods in Engineering, vol. 20, no. 3, pp. 167-182, 2004.

[10] M. K. Kadalbajoo and K. K. Sharma, "Numerical treatment of a mathematical model arising from a model of neuronal variability," Journal of Mathematical Analysis and Applications, vol. 307, no. 2, pp. 606-627, 2005.

[11] M. K. Kadalbajoo and D. Kumar, "Fitted mesh B-spline collocation method for singularly perturbed differential-difference equations with small delay," Applied Mathematics and Computation, vol. 204, no. 1, pp. 90-98, 2008.

[12] K. C. Patidar and K. K. Sharma, "Uniformly convergent nonstandard finite difference methods for singularly perturbed differential-difference equations with delay and advance," International Journal for Numerical Methods in Engineering, vol. 66, no. 2, pp. 272-296, 2006.

[13] M. K. Kadalbajoo, K. C. Patidar, and K. K. Sharma, "Uniformly convergent fitted methods for the numerical solution of the problems arising from singularly perturbed general DDEs," Applied Mathematics and Computation, vol. 182, no. 1, pp. 119139, 2006.

[14] V. Kumar and K. K. Sharma, "An optimized B-spline method for solving singularly perturbed differential difference equations with delay as well as advance," Neural, Parallel \& Scientific Computations, vol. 16, no. 3, pp. 371-385, 2008.

[15] L. E. El'sgol'ts and S. B. Norkin, Introduction to the Theory and Application of Differential Equations with Deviating Arguments, Academic Press, 1973.

[16] E. P. Doolan, J. J. H. Miller, and W. H. A. Schilders, Uniform Numerical Methods for Problems with Initial and Boundary Layers, Boole Press, Dublin, Ireland, 1980.

[17] R. E. O'Malley, Introduction to Singular Perturbations, Academic Press, New York, NY, USA, 1974.

[18] D. J. Evans, "Cage-Galerkin method for solving the 2-point boundary value problem," International Journal of Computer Mathematics, vol. 43, no. 3-4, pp. 219-230, 1992. 


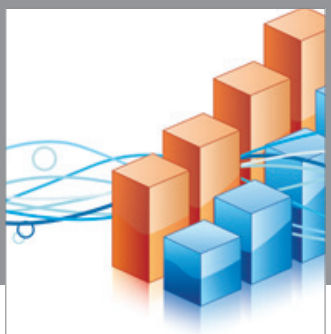

Advances in

Operations Research

vatem alat4

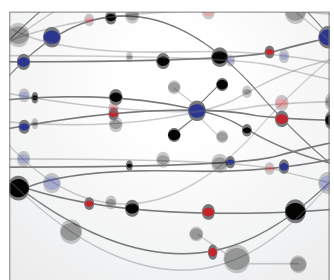

\section{The Scientific} World Journal
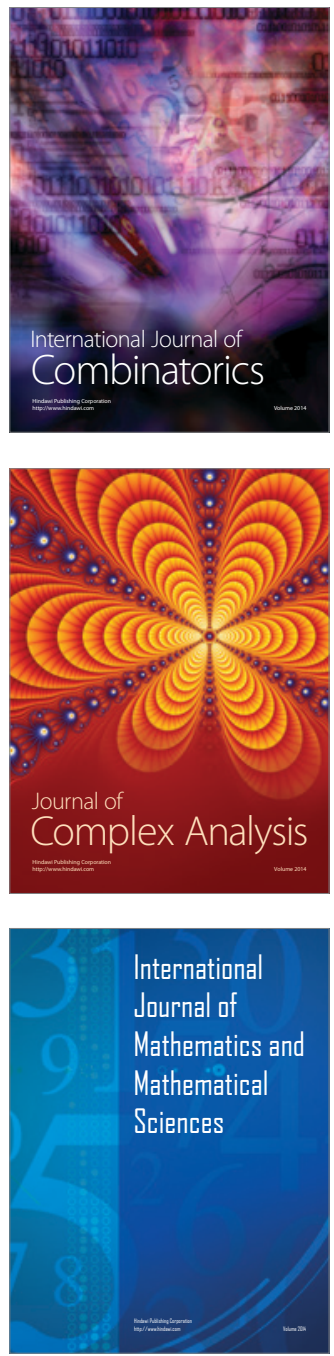
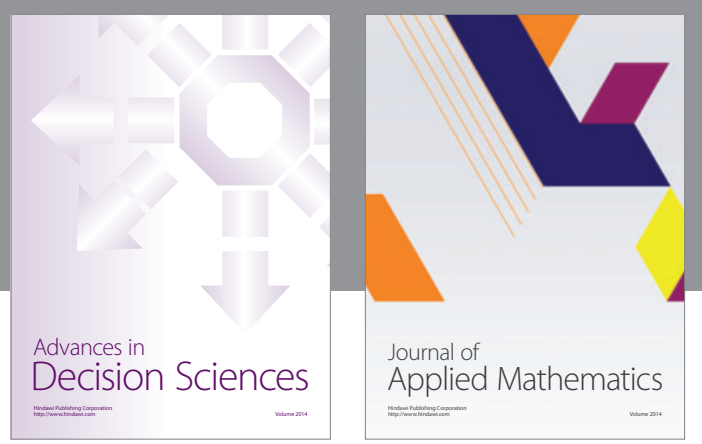

Algebra

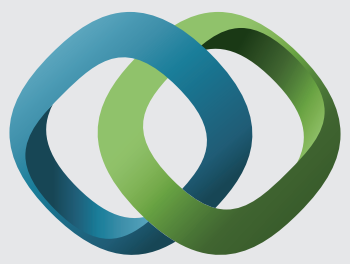

\section{Hindawi}

Submit your manuscripts at

http://www.hindawi.com
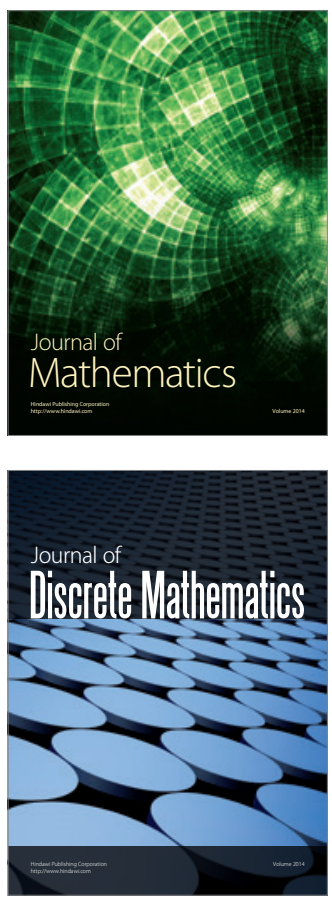

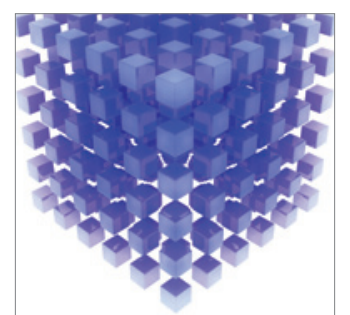

Mathematical Problems in Engineering
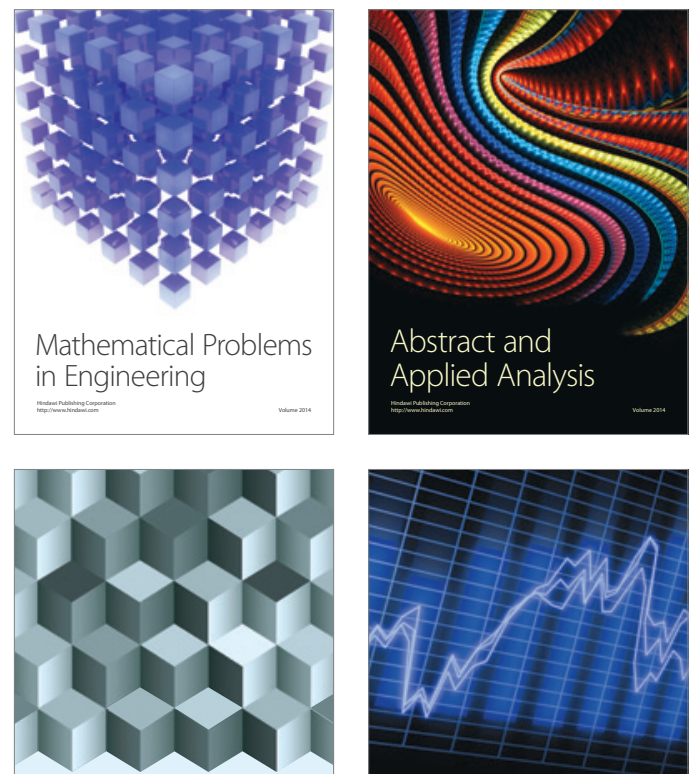

Journal of

Function Spaces

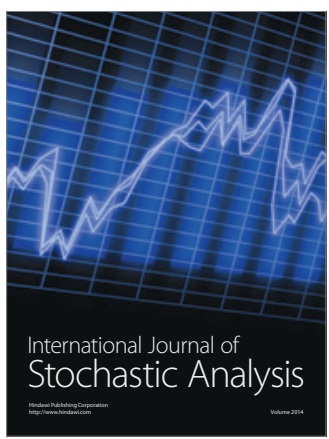

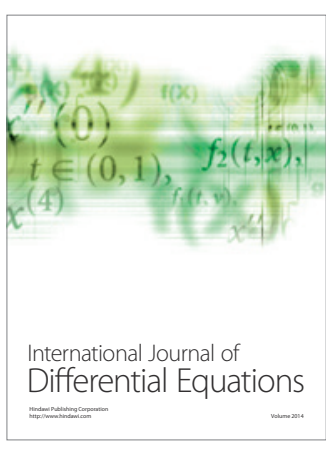
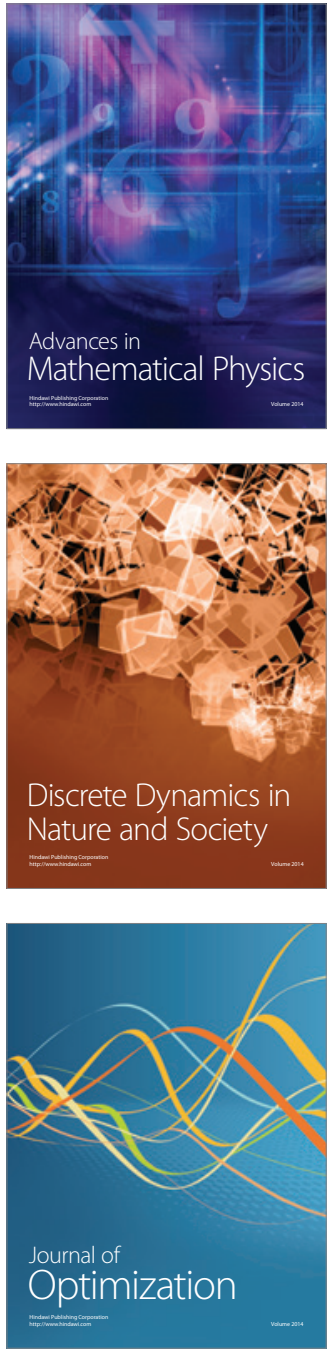